\title{
Dynamic Resource Allocation Scheme Using Cooperative Game for Multimedia Services in LTE Advanced System
}

\author{
Dapeng Wu \\ Chongqing University of Posts \\ and Telecommunications, \\ China \\ wudapengphd@gmail.com \\ Haisheng Yan \\ Chongqing University of Posts \\ and Telecommunications, \\ China \\ 530557803@qq.com
}

\author{
Xiaojun Zhou \\ Chongqing University of Posts \\ and Telecommunications, \\ China
}

\author{
Yi Xie \\ Chongqing telecom institute \\ fogartys@amesres.org
}

\author{
Ruyan Wang \\ Chongqing University of Posts \\ and Telecommunications, \\ China \\ wangry@cqupt.edu.cn
}

\begin{abstract}
In LTE Advanced system, it is critical to carry on various types of services for different user applications. Obviously, the system resource requirements are quite different for each individual service. In order to allocate and schedule limited resources reasonably, the cooperative game model is established and studied in our paper; furthermore, a novel scheme with dynamical system resource allocating and scheduling is also proposed, where the corresponding resources for each kind of service can be dynamically allocated according to the current traffic status. Consequently, the priorities of users are determined based on the service attribute and channel status. Results show that the spectral efficiency is enhanced effectively by our proposed scheme; in the mean time, the performances of multimedia services are also improved.
\end{abstract}

\section{Categories and Subject Descriptors}

C. 2 [COMPUTER-COMMUNICATION NETWORKS]: Miscellaneous

\section{General Terms}

Algorithms,Experimentation,Performance

\section{Keywords}

Long Term Evolution Advanced (LTE-A); cooperative game; resource allocation; resource scheduling

\section{INTRODUCTION}

Long Term Evolution Advanced (LTE-A) technologies have been studied by 3rd Generation Partnership Project (3GPP) since 2004 [1]. In LTE-A system, Orthogonal Frequency
Division Multiple Access (OFDMA) technology divides the frequency band into a group of mutually orthogonal subcarriers, so users can access and share the wireless medium at high data rate [2].

Obviously, to reasonably utilize the limited system resources is one of the most important challenges in LTE-A system. Currently, the service categories are expanded from the conventional voice service to hybrid services consisting of None Real Time (NRT) and Real Time (RT) services, such as voice, data, video et al. Similar to other kinds of networks, the demands on the system resources for various types of services vary, and the resources for RT services should be guaranteed to provide the satisfying Quality of Service (QoS) for users [3].

Currently, lots of resource allocating and scheduling mechanisms have been introduced to provide satisfying QoS for users. However, several key problems still remain unresolved. Firstly, the granularity of required system resources for individual service varies. Secondly, the applications are triggered and ceased by users randomly, so the resource requirements are changing dynamically. Moreover, the QoS demands for some applications cannot be satisfied due to the bursty of data packets.

In this paper, we propose a novel cooperative resource allocating and dynamical scheduling scheme which takes the feature of services into account. In order to meet the QoS demands for various services, system resources are allocated dynamically according to our proposed cooperative game model.

The remainder of this paper is organized as follows. In Section 2, some related works are surveyed, and the dynamic cooperative resource allocation scheme is proposed in Section 3. In Section 4, we evaluate the performance of our proposed scheme, and compare the proposed scheme with previous works. Finally, the conclusion and future works are given in Section 5.

\section{RELATED WORK}

In a LTE-A system, resource allocating and scheduling functions are implemented by the MAC layer scheduler of ENodeB [4]. During each Transmission Time Interval (TTI), the system resources are allocated and scheduled. The 
resources allocated to different user services are a series of resource blocks.

To guarantee the fairness of all the serving users, Proportional Fair (PF) algorithm has been introduced to perform the resource allocation in LTE-A systems [5]. Taking advantage of the inherent multi-user diversity, the system throughput can be improved. However, the delays of packets have not been considered.

In order to guarantee the delivery ratio and fairness of services, the delay of the head line and the maximum delay of a packet are considered together by Modified Largest Weighted Delay First (M-LWDF) algorithm [6]. The priority is determined by not only the instantaneous channel quality perceived by the user, but also the packet queuing delay, so the QoS of RT services can be guaranteed effectively.

To provide RT services for users, a QoS aware packet scheduler has been introduced in [7], where a novel two-level scheduling algorithm is designed. The upper level exploit$\mathrm{s}$ an innovative approach based on the discrete-time linear control theory. On the other hand, at the lower level, a proportional fair scheduler is designed. Results show that the proposed approach outperforms the existing ones especially in the presence of RT video flows.

Combining the virtual token mechanism with the cooperative game theory, a two level resource allocation scheme is proposed in [8] to enhance the QoS for multimedia services in LTE-A downlink system. By using the bankruptcy game and Shapley value, the proposed mechanism establishes coalitions between services in order to allocate resources fairly. In addition, the downlink packet scheduling algorith$\mathrm{m}$ is modified to use a virtual token mechanism to further improve the system performance.

According to the cooperative game theory, the network throughput improving mechanism is introduced in [9]. With the autonomous user collaboration, the access to the subchannel is decided. Consequently, resource allocation can be achieved by the distributed game method.

\section{DYNAMIC COOPERATIVE RESOURCE ALLOCATION}

In the hybrid multimedia service scenarios, system resources are shared by all kinds of flows, so the resource allocation for them is competitive; in the mean time, the system performance can be improved by allocating resources in the cooperative manner for services requiring various granularities. Therefore, the relationship of competition and cooperation for system resource allocation can be illustrated by the bankruptcy game model. The game model can be denoted by $G=(N, C, k)$, where $N=\{1,2, \cdots, n\}$ is the number of the service type, $C$ is the capacity of LTE-A system resources, $k=\left\{k_{1}, k_{2}, \cdots, k_{n}\right\}$ is the allocated resources for different services.

For different types of services, their transmission rates can be denoted by $b=\left\{b_{1}, b_{2}, \cdots, b_{n}\right\}$; furthermore, the corresponding numbers of certain services can be denoted by $g=\left\{g_{1}, g_{2}, \cdots, g_{n}\right\}$. As can be seen, the application resources for a given service are $k_{i}=g_{i} b_{i}$. According to the cooperative game theory, the proposed model $(N, C, k)$ can be simplified as $\left(N, v c_{k}\right)$, where $v c_{k}(S)$ denotes the minimum number of resources allocated to the flow set $S(1<|S|<N$ ) before the cooperation manner is employed, and it is shown in Eq. (1), $i \in N / S$ denotes $i \in N$ but $i \notin S$.

$$
v c_{k}(S)=\max \left\{C-\sum_{i \in N / S} k_{i}, 0\right\}
$$

The system resource allocation for LTE-A system, adopting the bankruptcy cooperative game model has three constraints: (1) individual rationality constraint; (2) integrated rationality constraint; (3) resource constant constraint. Al1 the constraints can be illustrated by Eq. (2), where $x_{i}$ is the resources allocated to the $i$ th type of service in the cooperation manner.

$$
\text { s.t. }\left\{\begin{array}{l}
x_{i} \geq v(i) \quad \forall i \in N \\
\sum_{i \in S} x_{i} \geq v(S) \quad \forall S \subset N \quad \text { and } \quad|\mathrm{S}|>1 \\
\sum_{i \in N} x_{i}=v(N)
\end{array}\right.
$$

As defined in the standard, there are three types of services in LTE-A system: video, Voice over Internet Protocol (VoIP) and Constant Bit Rate (CBR) services respectively. Under the cooperative game model, the set of service types can be denoted as $N=\left\{n_{1}, n_{2}, n_{3}\right\}$, and the obtained system resources for all kinds of services after the cooperative allocation are $x=\left\{x_{n 1}, x_{n 2}, x_{n 3}\right\}$. According to Eq. (1), the minimum number of system resources allocated can be evaluated as shown in Eq. (3), where $v\left(n_{i}\right)$ is the minimum number of resources allocated for service $n_{i}$ without the cooperation method, $v\left(n_{i}, n_{j}\right)$ is the minimum number of resources allocated for the service set which consists of $n_{i}$ and $n_{j}$ without the cooperation method.

$$
\left\{\begin{array}{l}
v\left(n_{i}\right)=\max \left\{C-\sum_{j \in N, j \neq i} b_{n j} g_{n j}, 0\right\} \\
v\left(n_{1}, n_{2}\right)=\max \left\{C-b_{3} g_{3}, 0\right\} \\
v\left(n_{1}, n_{3}\right)=\max \left\{C-b_{2} g_{2}, 0\right\} \\
v\left(n_{2}, n_{3}\right)=\max \left\{C-b_{1} g_{1}, 0\right\} \\
v\left(n_{1}, n_{2}, n_{3}\right)=C
\end{array}\right.
$$

Obviously, the required resources under different kinds of service sets should meet the constraints shown in Eq. (2). There is a certain probability that the service will be allocated with more resources under local cooperation than the case of global cooperation, where the local cooperation means that only a part of services are from the cooperation, and the global cooperation means that the cooperation involves all the services. As a result, the set $x=\left\{x_{n 1}, x_{n 2}, x_{n 3}\right\}$ is empty. To solve this problem, a slack variable $\varepsilon$ is added to the integrated consistency constraint. And the resources to be allocated for different kinds of services can be obtained from Eq. (4).

$$
\left\{\begin{array}{l}
x_{n 1}+x_{n 2}=v\left(n_{1}, n_{2}\right)+\varepsilon_{1} \\
x_{n 1}+x_{n 3}=v\left(n_{1}, n_{3}\right)+\varepsilon_{2} \\
x_{n 2}+x_{n 3}=v\left(n_{2}, n_{3}\right)+\varepsilon_{3} \\
x_{n 1}+x_{n 2}+x_{n 3}=v\left(n_{1}, n_{2}, n_{3}\right)
\end{array}\right.
$$

According to our model, the application resources for $\mathrm{d}$ ifferent kinds of services should meet the constraint $k_{n 1}$ : $k_{n 2}: k_{n 3}=b_{n 1} g_{n 1}: b_{n 2} g_{n 2}: b_{n 3} g_{n 3}$, so the slack variables can be set as $\varepsilon_{1}=k_{n 3} \varepsilon, \varepsilon_{2}=k_{n 2} \varepsilon, \varepsilon_{3}=k_{n 1} \varepsilon$. Combining Eq. (4), the value of $\varepsilon$ can be obtained from Eq.(5).

$$
\varepsilon=\frac{2 v\left(n_{1}, n_{2}, n_{3}\right)-\left[v\left(n_{1}, n_{2}\right)+v\left(n_{1}, n_{3}\right)+v\left(n_{2}, n_{3}\right)\right]}{\left(b_{n 1} g_{n 1}+b_{n 2} g_{n 2}+b_{n 3} g_{n 3}\right)}
$$


With the cooperation method, the resources to be allocated for services are $x_{n 1}, x_{n 2}$ and $x_{n 3}$. Consequently, the relationship between the resources allocated to the video service and the slack variable can be described by Eq. (6).

$$
x_{n 1}=v\left(n_{1}, n_{2}, n_{3}\right)-v\left(n_{2}, n_{3}\right)-b_{n 1} g_{n 1} \varepsilon
$$

Consequently, the relationship between resources and slack variables for other types of services can be obtained.

As mentioned above, the basic granularity of the allocated resources in LTE-A system is PRB. According to our cooperative game model, the residual resources may exist in a practical system. Obviously, by allocating the residual resources reasonably, the system performance can be further improved.

According to the service feature defined in the standard, RT services and NRT services should both be supported simultaneously. Further, while scheduling resources for NRT traffic, the channel conditions, fairness and other factors should be considered comprehensively; but in RT traffic scheduling process, the head buffering delay and the maximum packet delay should be taken into account primarily. In traditional resource allocating and scheduling schemes, resources are always assigned to the user with the highest priority. Before a resource scheduling scheme executes, ENodeB computes the scheduling priority of each user first, and then the corresponding resources are allocated to a given user until all the system resources are exhausted. While the resources for a certain service are allocated completely, the scheduling priority for corresponding PRBs is set to zero.

\section{NUMERICAL RESULTS}

\subsection{Simulation Setup}

In this section, our proposed resource allocating and scheduling scheme is simulated [10]. Similar to [11], the proportion of the flow number for each kind of service is set to $2: 2: 1$; additionally, other related parameters are listed in Table 2.To model the service of VoIP, we assume that VoIP application generates G.729 voice flows.

Table 1: Parameter Setting

\begin{tabular}{lc}
\hline Parameter & Value \\
\hline Radius & $1 \mathrm{Km}$ \\
Bandwidth & $10 \mathrm{MHz}$ \\
Scheduling time & $1 \mathrm{~ms}$ \\
Capacity & $32000 \mathrm{kbps}$ \\
Number of PRBs & 50 \\
Frame structure & FDD \\
Max delay & $100 \mathrm{~ms}$ \\
Video bit-rate & $242 \mathrm{kbps}$ \\
VoIP bit-rate & $8 \mathrm{kbps}$ \\
Mobile Speed & $0-3 \mathrm{Km} / \mathrm{h}$ \\
Multi-path Model & Jakes Model \\
\hline
\end{tabular}

\subsection{Results}

In this section, the performance of our proposed Dynamical Resource Allocating and Scheduling scheme using Cooperative Game (DRAS-CG) model is compared with a similar strategy called DAPS, which was introduced in [12]. In addition, some traditional schemes are also evaluated, such as M-LWDF and EXP-RULE.

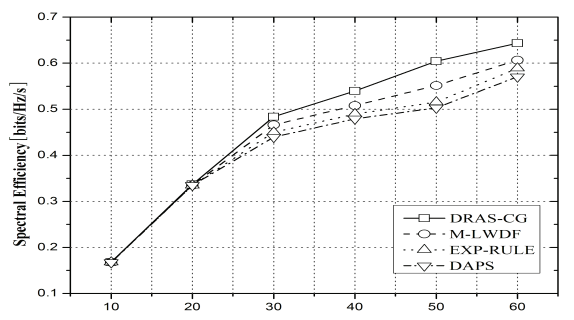

Figure 1: Spectral Efficiency

The spectral efficiency of different resource allocation methods is shown in Figure 1. Results show that, when the number of users is 60, the spectral efficiency of DRAS-CG is $9 \%$ higher than that of DAPS.

The average throughput of the video service is shown in Figure 2. Results show that the average throughput of the video service decreases with the increasing number of users, and the average throughput of DRAS-CG is the highest among all four schemes. When the number of users is 60 , the average throughput of DRAS-CG is $10 \%$ higher than those of the other three schemes. The packet loss ratio of the video service is shown in Figure 3. It can be seen that the packet loss ratio increases along with the number of users, and the packet loss ratio of DRAS-CG is $21 \%$ lower than those of the other three schemes. The performance of the average delay is shown in Figure 4, when the delay of a packet is larger than $50 \mathrm{~ms}$, it will be dropped by all the schedulers due to the pre-defined delay threshold. While the user number is 50 , the delay of DRAS-CG is $14 \%$ lower than those of others. Finally, the performance of the fairness is shown in Figure 5 , while the user number is small, the performances of all four schemes are similar; however, in the cases of larger user numbers, such as 50 or 60 , the gain of our proposed scheme becomes larger.
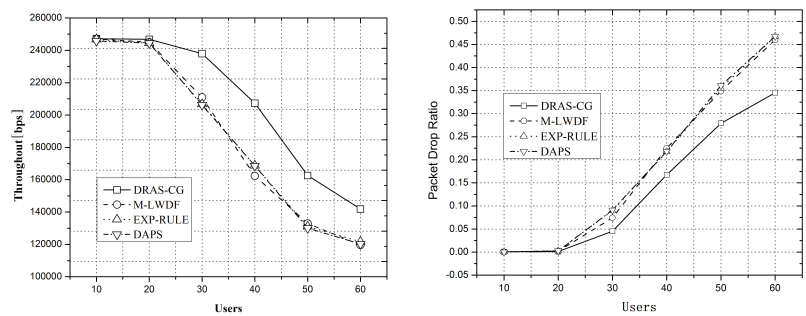

Figure 2: Throughput ofFigure 3: Packet Drop Ratio Video Service of Video Service
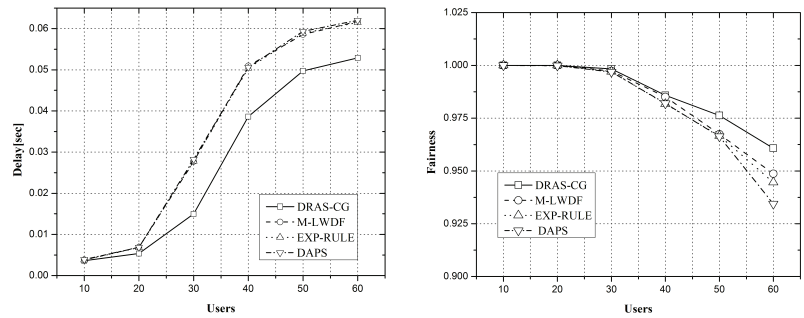

Figure 4: Delay of Video Ser-Figure 5: Fairness of Video vice Service

The performances of VoIP service are shown in Figures 6 to 9 . From the results, the throughputs of voice service for different schemes are close, and they fluctuate within a small range. The packet loss ratio of VoIP is shown in Figure 7, where the performances for different schemes are similar when the number of users is less than 40. As the 
number of users increases continuously, the packet loss ratio of DRAS-CG increases slowly, and the packet loss ratios of other schemes increase faster. As the number of users increases, the delays of VoIP become larger for all the schemes. When the number of users is 60, the delay of DRAS-CG is $35 \%$ lower than those of other resource allocation schemes. Similar with the video service performance, the fairness of VoIP service for these four schemes are identical.
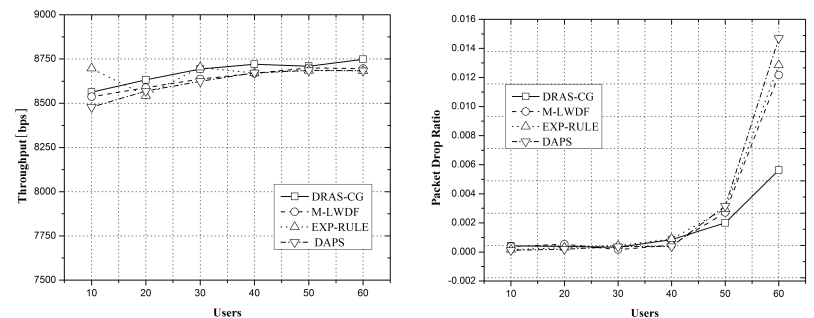

Figure 6: Throughput of VoIPFigure 7: Packet Drop Ratio Service of VoIP Service
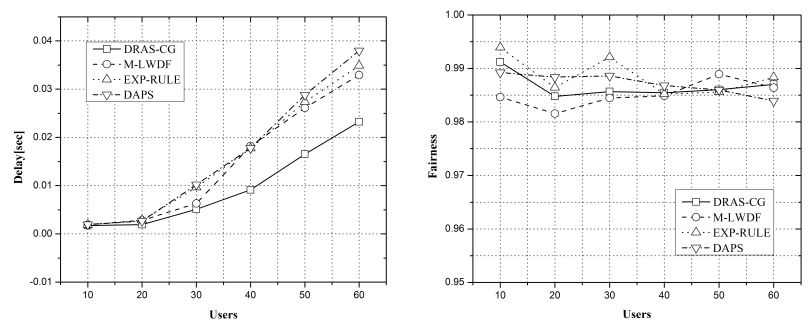

Figure 8: Delay of VoIP Ser-Figure 9: Fairness of VoIP vice Service

\section{CONCLUSIONS}

With the rapid development of wireless multimedia services, it becomes the top priority to optimize the limited system resources. A dynamical resource allocating and scheduling scheme based on cooperative game theory is proposed in this paper. According to the service attributes, system resources and resource constraints, the physical resources are allocated dynamically to each kind of service. Our results show that the QoS of real time service can be implemented more effectively by the proposed method; in the mean time, the spectral efficiency of the system can be improved dramatically. Our future work will mainly focus on the dynamical determination of urgent flows; on the other hand, the QoE (Quality of Experience) aware resource allocation strategy will also need to be studied. 1

\section{ACKNOWLEDGMENTS}

This work is supported in part by the National Natural Science Foundation of China (61371097), Chongqing Natural Science Foundation (Grant No. CSTC2013JJB40001, CSTC2013JJB40006, CSTC2011JJA40043, CSTC2014JCYJA 40039), the Project of Chongqing Education commission (KJ1400402), the Foundation of Chongqing University of Posts and Telecommunication (A2012-93), Youth Talents Training Project of Chongqing Science \& Technology Commission (cstc2014kjrc-qnrc40001).

\section{REFERENCES}

[1] C. M. Lim, T. S. Yoo, B. Clerckx, et al, "Recent trend of multiuser MIMO in LTE-advanced," IEEE
Communications Magazine, vol. 51, no. 3, pp. 127-135, Mar. 2013.

[2] Z. Zhang, J. Zhang, S. Sun. "Model of Handover and Traffic Based on Cellular Geometry with Smart Antenna," International Journal of Antennas and Propagation, vol. 2014, Article ID 646053, Apr. 2014.

[3] B. S. Abdallah, R. Simone, R. Bernhard, "Resource sharing in LTE-Advanced relay networks: uplink system performance analysis," Transactions on Emerging Telecommunications Technologies, vol. 24, no. 1, pp. 32-48, Jan. 2013.

[4] C. Khirallah, D. Vukobratovic, J. Thompson, "Performance Analysis and Energy Efficiency of Random Network Coding in LTE-Advanced," IEEE Transactions on Wireless Communications, vol. 11, no. 12, pp. 4275-4285, Dec. 2012.

[5] G. J. Choi, "Cell-throughput analysis of the proportional fair scheduler in the single-cell environment," IEEE Transactions on Vehicular Technology, vol. 56, no. 2, pp. 766-778, Mar.2007.

[6] P. Ameigeiras, J. Wigard, P. Mogensen, "Performance of the M-LWDF scheduling algorithm for streaming services in HSDPA," in Proc. 60th IEEE Vehicular Technology Conference (VTC), Sep. 26-29, 2004, pp. 999-1003.

[7] G. Piro, L. Grieco, G. Boggia, F. Capozzi, et al, "Two-Level Downlink Scheduling for Real-Time Multimedia Services in LTE Networks," IEEE Transactions on Multimedia, vol. 13, no. 5, pp. 1052-1065, May 2011.

[8] M. Iturralde, A. Wei, T. Yahiya, et al, "Resource Allocation for Real Time Services in LTE Networks: Resource Allocation Using Cooperative Game Theory and Virtual Token Mechanism," Wireless Personal Communications, vol. 72, no. 2, pp. 1-21, Sep. 2013.

[9] O. N. Gharehshiran, A. Attar, V. Krishnamurthy, "Collaborative Sub-Channel Allocation in Cognitive LTE Femto-Cells: A Cooperative Game-Theoretic Approach," IEEE Transactions on Communications, vol. 61 , no. 1, pp. 325-334, Jan. 2013.

[10] G. Piro, L. Grieco, G. Boggia, F. Capiozzi, et al, "Simulating LTE cellular systems: an open source framework," IEEE Transaction on Vehicular Technology, vol. 60, no. 2, pp. 498-513, Feb. 2011.

[11] M. Iturralde, T. Yahiya, A. Wei, et al, "Performance Study of Multimedia Services Using Virtual Token Mechanism for Resource Allocation in LTE Networks," in Proc. IEEE Vehicular Technology Conference (VTC Fall), San Francisco, CA, USA, Sep. 5-8, 2011, pp. 1-5.

[12] B. G. Choi, J. B. Sueng, K. R. Jung, et al, "Resource Allocation Scheme Considering Local Channel Quality Information and Buffer Status of Relay Nodes in Relay-Based Cellular Networks," IEEE Communications Letters, vol. 15, no. 12, pp. 1304-1306, Dec. 2011. 